\title{
EXTREME INVARIANT POSITIVE OPERATORS ON $L_{p}$-SPACES
}

\author{
HARALD LUSCHGY
}

\begin{abstract}
Let $(X, \mathfrak{A}, \mu)$ and $(Y, \mathfrak{B}, \nu)$ be finite positive measure spaces. In this note we present characterizations of the extreme points of the convex set of all positive linear operators $T: L_{p}(\mu) \rightarrow L_{q}(\nu)$ with $T 1_{X}=1_{Y}$ which are invariant with respect to a semigroup of positive constant preserving contractions on $L_{p}(\mu), 1 \leqslant p<\infty, 1 \leqslant q<\infty$.
\end{abstract}

Introduction. Let $(X, \mathfrak{A}, \mu)$ and $(Y, \mathfrak{B}, \nu)$ be finite positive measure spaces and let $K\left[L_{p}(\mu), L_{q}(\nu)\right]$ denote the convex set of all positive linear operators $T: L_{p}(\mu) \rightarrow L_{q}(\nu)$ with $T \mathbf{1}_{X}=\mathbf{1}_{Y}$ for $1 \leqslant p \leqslant \infty, 1 \leqslant q \leqslant \infty$. It is known that an operator $T$ in $K\left[L_{p}(\mu), L_{q}(\nu)\right]$ is an extreme point of this set if and only if $T$ is a lattice homomorphism [6, III.9.2]. Further characterizations of the extreme points of $K\left[L_{\infty}(\mu), L_{\infty}(\nu)\right]$ as operators which are multiplicative or which carry characteristic functions into characteristic functions are given by Phelps $[5,2.2]$. We will characterize the extreme points of the set $K\left[L_{p}(\mu)\right.$, $\left.L_{q}(\nu)\right]_{G}$ of all operators in $K\left[L_{p}(\mu), L_{q}(\nu)\right]$ which are invariant with respect to a semigroup $G$ of positive constant preserving contractions on $L_{p}(\mu)$ for $p<\infty$. These characterizations, including generalizations of the above mentioned results, are in part similar to those of the extreme points of $K[C(X), C(Y)]_{G}$ for compact spaces $X$ and $Y$, which have been stated by Converse, Namioka and Phelps [2, 5.3].

1. Preliminaries. Throughout suppose that $(X, \mathfrak{A}, \mu)$ and $(Y, \mathfrak{B}, \nu)$ are finite positive measure spaces. $\mathbf{1}_{A}$ stands for the characteristic function of $A$. The convex set of all positive linear operators $T: L_{p}(\mu) \rightarrow L_{q}(\nu)$ with $T \mathbf{1}_{X}=\mathbf{1}_{Y}$ is denoted by $K\left[L_{p}(\mu), L_{q}(\nu)\right]$ for $1 \leqslant p \leqslant \infty, 1 \leqslant q \leqslant \infty$. Let $G$ be a subsemigroup of $K\left[L_{p}(\mu), L_{p}(\mu)\right]$. A linear operator $T: L_{p}(\mu) \rightarrow L_{q}(\nu)$ is called invariant if $T V=T$ for all $V \in G . K\left[L_{p}(\mu), L_{q}(\nu)\right]_{G}$ denotes the convex set of all invariant elements in $K\left[L_{p}(\mu), L_{q}(\nu)\right]$. Furthermore, we denote by $D$ the linear hull of the set $\left\{V f-f: f \in L_{p}(\mu), V \in G\right\}$ and by $F$ the fixed space of $G$, i.e. $F=\left\{f \in L_{p}(\mu): V f=f\right.$ for all $\left.V \in G\right\}$.

The key for the characterizations of extreme points is the following fact. If $G$ is a contractive semigroup, i.e. $\sup \{\|V\|: V \in G\} \leqslant 1$ and $p<\infty$, then the semigroup $\operatorname{co}(G)^{-}$has a zero element, where $\operatorname{co}(G)^{-}$denotes the closed convex hull of $G$ in the space of all continuous linear operators on $L_{p}(\mu)$,

Received by the editors December 12, 1977.

AMS (MOS) subject classifications (1970). Primary 46E30, 47D20; Secondary 47D05.

$K e y$ words and phrases. $L_{p}$-spaces, extreme invariant positive linear operators, contractive semigroups, conditional expectations.

(๑) American Mathematical Society 1978 
endowed with the topology of simple convergence [4, 1.4 and 2.3]. The (unique) zero element $P$ of $\operatorname{co}(G)^{-}$is a positive contractive projection onto $F$ with $P 1_{X}=1_{X}$ (cf. [6, III.7.2]). Furthermore, an operator $T \in$ $K\left[L_{p}(\mu), L_{q}(\nu)\right]$ is invariant if and only if $T P=T$. This follows from the continuity of $T$ (cf. [6, II.5.3]).

2. Extreme invariant operators. The following characterization, which holds without any further hypotheses on $G$ and $p$, is a special case of $[3$, Theorem 5].

Theorem 1. Suppose $T \in K\left[L_{p}(\mu), L_{q}(\nu)\right]_{G}$. Then $T$ is an extreme point of $K\left[L_{p}(\mu), L_{q}(\nu)\right]_{G}$ if and only if $\inf \left\{T(|f-h|): h \in R \mathbf{1}_{X}+D\right\}=0$ for each $f \in L_{p}(\mu)$.

Before we can formulate the main result, we need the following information.

LEMMA. If $G$ is a contractive semigroup, $p<\infty$, and $P$ is the zero element of $\operatorname{co}(G)^{-}$, then $F=L_{p}\left(\mu \mid \mathfrak{A}_{0}\right)$ and $P$ is the $\mathfrak{U}_{0}$-conditional expectation with $\mathfrak{U}_{0}=\left\{A \in \mathfrak{U}: \mathbf{1}_{A} \in F\right\}$.

Proof. Obviously $F$ is a closed subspace of $L_{p}(\mu)$ with $\mathbf{1}_{X} \in F$. Furthermore, $F$ is a sublattice. Let $f \in F$ and $V \in G$. Since $f^{+} \geqslant f$ and $f^{+} \geqslant 0$ we have $V f^{+} \geqslant V f=f$ and $V f^{+} \geqslant 0$. Hence, $V f^{+} \geqslant f^{+}$and this implies $V f^{+}=$ $f^{+}$because $V$ is a contraction. The first assertion follows from the wellknown characterization of closed sublattices of $L_{p}(\mu)$ which contain $\mathbf{1}_{X}$ (cf. [6, III.11.2]). In view of the above mentioned properties of $P$, the second assertion is a result of Ando [1, (proof of) Theorem 2].

Let $E_{\mathfrak{A}_{0}}$ denote the $\mathfrak{U}_{0}$-conditional expectation operator.

Theorem 2. Suppose $T \in K\left[L_{p}(\mu), L_{q}(\nu)\right]_{G}$ and $p<\infty$. If $G$ is a contractive semigroup, then the following assertions are equivalent.

(i) $T$ is an extreme point of $K\left[L_{p}(\mu), L_{q}(\nu)\right]_{G}$.

(ii) $\inf \left\{T\left(\left|f-t \mathbf{1}_{X}\right|\right): t \in R\right\}=0$ for each $f \in F$.

(iii) $T\left(E_{\mathfrak{A}_{0}} f \cdot h\right)=T f T h$ for each $f \in L_{p}(\mu), h \in L_{\infty}(\mu)$.

(iv) $T($ fh $)=T f T h$ for each $f \in F, h \in L_{\infty}\left(\mu \mid \mathfrak{A}_{0}\right)$.

(v) $T \mid F$ is a lattice homomorphism.

(vi) $T$ carries $\mathfrak{A}_{0}$-measurable characteristic functions into characteristic functions.

Proof. The equivalence of (i) and (ii) follows from [3, Theorem 6].

(i) $\Rightarrow$ (iii). Clearly it is sufficient to prove that assertion (iii) holds for those $h \in L_{\infty}(\mu)$ such that $0 \leqslant h \leqslant 1_{X}$. Assuming $0 \leqslant h \leqslant \mathbf{1}_{X}$, we define a map $T_{0}: L_{p}(\mu) \rightarrow L_{q}(\nu)$ by $T_{0} f:=T\left(E_{\mathfrak{A}_{0}} f \cdot h\right)-T f T h$. Then $T_{0}$ is an invariant linear operator with $T_{0} \mathbf{1}_{X}=0$. If $f \geqslant 0$, then $\left(T+T_{0}\right) f=T f\left(1_{Y}-T h\right)+$ $T\left(E_{\mathscr{Q}_{0}} f \cdot h\right) \geqslant 0$ and $\left(T-T_{0}\right) f=T\left[E_{\mathfrak{Q}_{0}} f \cdot\left(1_{X}-h\right)\right]+T f T h \geqslant 0$. Thus $T$ $\pm T_{0} \in K\left[L_{p}(\mu), L_{q}(\nu)\right]_{G}$ holds. Since $T$ is extreme, this implies $T_{0}=0$.

(iii) $\Rightarrow$ (iv) is obvious. 
(iv) $\Rightarrow(\mathrm{v})$. First let $f \in L_{\infty}\left(\mu \mid \mathfrak{A}_{0}\right)$. The chain $(T|f|)^{2}=T\left(|f|^{2}\right)=T\left(f^{2}\right)=$ $(T f)^{2}=|T f|^{2}$ shows that $|T f|=T|f|$ holds. Since $L_{\infty}\left(\mu \mid \mathfrak{A}_{0}\right)$ is dense in $F$ and furthermore, $T$ and the lattice operations on $L_{p}(\mu)$ and $L_{q}(\nu)$ are continuous, this implies that $T \mid F$ is a lattice homomorphism.

(v) $\Rightarrow$ (vi). For $A \in \mathfrak{A}_{0}$ we obtain $T 1_{A} \wedge T 1_{A^{c}}=T\left(1_{A} \wedge 1_{A^{c}}\right)=T 0=0$. Hence, $T \mathbf{1}_{A}$ is a characteristic function.

(vi) $\Rightarrow$ (ii). Let $A \in \mathfrak{A}_{0}$. Since $T\left(\left|\mathbf{1}_{A}-t \mathbf{1}_{X}\right|\right)=|1-t| T \mathbf{1}_{A}+|t| T \mathbf{1}_{A}$, it follows that

$$
\inf \left\{T\left(\left|\mathbf{1}_{A}-t \mathbf{1}_{X}\right|\right): t \in R\right\} \leqslant T \mathbf{1}_{A} \wedge T \mathbf{1}_{A^{c}}=0 .
$$

By virtue of the continuity of $T$ it is readily verified that $\inf \left\{T\left(\left|f-t \mathbf{1}_{X}\right|\right)\right.$ : $t \in R\}=0$ is valid for each $f \in F$.

CoRollary 1. Suppose $T \in K\left[L_{p}(\mu), R\right]_{G}$. Under the above hypotheses on $G$ and $p, T$ is an extreme point of $K\left[L_{p}(\mu), R\right]_{G}$ if and only if $T \mathbf{1}_{A} \in\{0,1\}$ for each $A \in \mathfrak{A}_{0}$.

In the following corollary an application to conditional expectations is given.

CoRollary 2. Suppose that $\mathfrak{A}_{1}$ is a $\sigma$-subalgebra of $\mathfrak{A}_{0}$. Under the above hypotheses on $G$ and $p$, the operator $E_{\mathfrak{A}_{1}}$ is an extreme point of $K\left[L_{p}(\mu), L_{p}(\mu)\right]_{G}$ if and only if for each $A \in \mathfrak{U}_{0}$ there exists $B \in \mathfrak{A}_{1}$ with $\mu(A \triangle B)=0$.

Proof. Obviously $E_{\mathfrak{Y}_{1}} \in K\left[L_{p}(\mu), L_{p}(\mu)\right]_{G}$ holds.

The "if" part. Let $A \in \mathfrak{A}_{0}$. By assumption there exists $B \in \mathfrak{A}_{1}$ with $\mu(A \triangle B)=0$ such that $E_{\mathscr{Q}_{1}} \mathbf{1}_{A}=E_{\mathfrak{Q}_{1}} \mathbf{1}_{B}=\mathbf{1}_{B}$. The assertion follows from Theorem 2 .

The "only if" part. Let $A \in \mathfrak{A}_{0}$. From Theorem 2 follows

$$
\begin{aligned}
\mu(A \cap C) & =\int E_{\mathscr{U}_{1}}\left(\mathbf{1}_{A} \mathbf{1}_{C}\right) d \mu \\
& =\int E_{\mathfrak{Q}_{1}} \mathbf{1}_{A} E_{\mathfrak{Y}_{1}} \mathbf{1}_{C} d \mu=\int \mathbf{1}_{C} E_{\mathfrak{Y}_{1}} \mathbf{1}_{A} d \mu
\end{aligned}
$$

for each $C \in \mathfrak{A}_{0}$. Hence, $E_{\mathfrak{U}_{1}} \mathbf{1}_{A}=\mathbf{1}_{A}$. This yields the assertion.

Remark. Let $G \subset K\left[L_{\infty}(\mu), L_{\infty}(\mu)\right]$. Then $V \in G$ can be extended to a positive linear contraction on $L_{1}(\mu)$ if (and only if) $V$ is expectation invariant, i.e. $\int V f d \mu=\int f d \mu$ for each $f \in L_{\infty}(\mu)$. Thus the preceding characterizations are valid for $p=\infty$ if $G$ is a semigroup of expectation invariant operators.

\section{REFERENCES}

1. T. Ando, Contractive projections in $L_{p}$-spaces, Pacific J. Math. 17 (1966), 391-405. MR 33 \#566.

2. G. Converse, I. Namioka and R. R. Phelps, Extreme invariant positive operators, Trans. Amer. Math. Soc. 137 (1969), 375-385. MR 39 \# 4692. 
3. H. Luschgy, Invariant extensions of positive operators and extreme points, Trans. Amer. Math. Soc. (submitted).

4. R. J. Nagel, Mittelergodische Halbgruppen linearer Operatoren, Ann. Inst. Fourier 23 (1973), 75-87. MR 49 \# 11283.

5. R. R. Phelps, Extreme positive operators and homomorphisms, Trans. Amer. Math. Soc. 108 (1963), 265-274. MR 27 \#6153.

6. H. H. Schaefer, Banach lattices and positive operators, Springer-Verlag, Berlin and New York, 1974.

Stiftsherrenstrasse 5, D-4400 Münster/Westfalen, Federal Republic of Grrmany 